Supporting Information for

\title{
Protecting enzymes from stress-induced inactivation
}

Samantha Piszkiewicz ${ }^{\dagger}$ and Gary J. Pielak ${ }^{\dagger, \ddagger, \S, \text { * }}$

†Department of Chemistry

¥Department of Biochemistry and Biophysics

§Lineberger Comprehensive Cancer Center

"Integrative Program for Biological and Genome Sciences University of North Carolina, Chapel Hill, NC, USA 27599

*To whom correspondence should be addressed. Tel: 9199624495 E-mail: gary_pielak@unc.edu.

Tables: 3

Figures: 0

Abbreviations: bovine serum albumin, BSA; 3-[(3-cholamidopropyl)-dimethylammonio]-1propanesulfonate, CHAPS; cytosolic abundant heat soluble, CAHS; lactate dehydrogenase, LDH; late embryogenesis abundant, LEA; red fluorescent protein, mCherry; polyethylene glycol, PEG; sodium dodecyl sulfate, SDS; trimethylamine-Noxide, TMAO. 
Table S1. Ability of additives to protect proteins against vacuum-drying

\begin{tabular}{|c|c|c|c|c|}
\hline Test Protein & Assay & Output & Additive & Protection \\
\hline $\begin{array}{l}\text { ADP-glucose- } \\
\text { pyrophosphorylase }\end{array}$ & activity & absorbance & $\begin{array}{l}\beta \text {-lactoglobulin } \\
\text { LEA protein } \\
\text { ribonuclease A }\end{array}$ & $\begin{array}{l}\text { none }^{1} \\
\text { partial }^{1} \\
\text { none }^{1}\end{array}$ \\
\hline $\begin{array}{c}\text { Aphelenchus } \\
\text { avenae proteome }\end{array}$ & aggregation & light scattering & LEA protein & full ${ }^{2}$ \\
\hline BamHI & activity & DNA digestion & trehalose & partial $^{3}$ \\
\hline Bglll & activity & DNA digestion & trehalose $^{a}$ & partial $^{4}$ \\
\hline citrate synthase & $\begin{array}{l}\text { activity } \\
\text { aggregation }\end{array}$ & $\begin{array}{l}\text { absorbance } \\
\text { light scattering }\end{array}$ & $\begin{array}{l}\text { BSA } \\
\text { LEA protein } \\
\text { BSA } \\
\text { HSP70 } \\
\text { LEA protein }\end{array}$ & $\begin{array}{l}\text { partial }^{5} \\
\text { full }^{5} \\
\text { none }^{5} \\
\text { none } \\
\text { full }{ }^{5,6}\end{array}$ \\
\hline EcoRI & activity & DNA digestion & $\begin{array}{l}\text { maltodextrin } \\
\text { polyvinylpyrrolidone } \\
\text { raffinose } \\
\text { sucrose } \\
\text { trehalose }^{a}\end{array}$ & $\begin{array}{l}\text { partial }^{7} \\
\text { partial }^{7} \\
\text { partial }^{7} \\
\text { partial }^{7} \\
\text { partial }^{3,4,7}\end{array}$ \\
\hline fumarase & activity & activity & $\begin{array}{l}\text { BSA } \\
\text { LEA protein }\end{array}$ & $\begin{array}{l}\text { none }^{8} \\
\text { partial }^{8}\end{array}$ \\
\hline $\begin{array}{l}\text { glucose-6- } \\
\text { phospho- } \\
\text { dehydrogenase }\end{array}$ & activity & absorbance & $\begin{array}{l}\text { } \beta \text {-lactoglobulin } \\
\text { LEA protein } \\
\text { ribonuclease A }\end{array}$ & $\begin{array}{l}\text { partial }^{1} \\
\text { partial }^{1} \\
\text { none }^{1}\end{array}$ \\
\hline
\end{tabular}




\begin{tabular}{|c|c|c|c|c|}
\hline Test Protein & Assay & Output & Additive & Protection \\
\hline HindIII & activity & DNA digestion & $\begin{array}{l}\text { maltose } \\
\text { sucrose } \\
\text { trehalose }\end{array}$ & $\begin{array}{l}\text { partial }^{3} \\
\text { partial }^{3} \\
\text { partial }^{3}\end{array}$ \\
\hline $\begin{array}{c}\text { lactate } \\
\text { dehydorgenase }\end{array}$ & activity & absorbance & 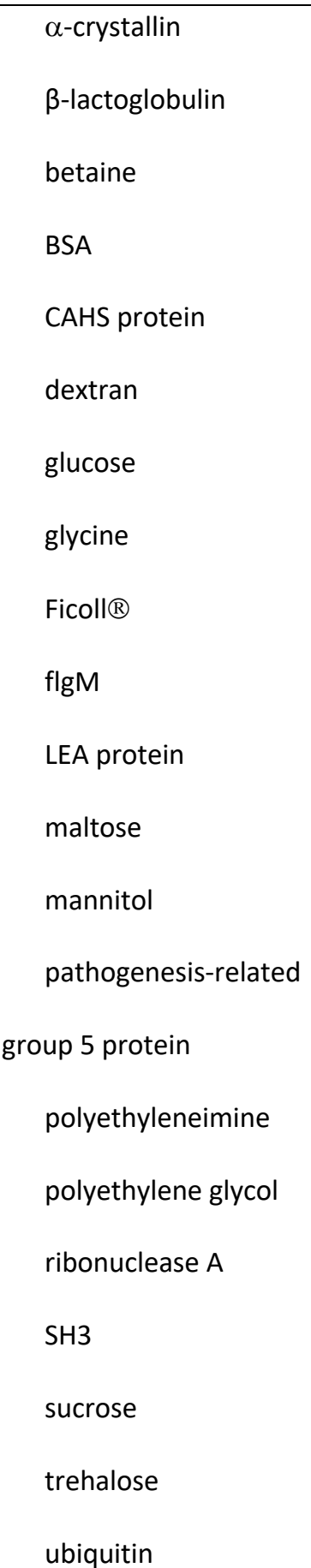 & $\begin{array}{l}\text { partial }^{9} \\
\text { partial }^{1} \\
\text { none }^{10} \\
\text { full, }^{10,11} \text { partial }^{5,9,12} \\
\text { full }^{10,11} \\
\text { partial }^{10} \\
\text { none }{ }^{10} \\
\text { none }{ }^{10} \\
\text { full }^{10} \\
\text { full }^{10} \\
\text { partial }^{1,5,9,12} \\
\text { partial }^{10} \\
\text { partial }^{10} \\
\text { none }\end{array}$ \\
\hline
\end{tabular}




\begin{tabular}{|c|c|c|c|c|}
\hline Test Protein & Assay & Output & Additive & Protection \\
\hline mCherry & $\begin{array}{l}\text { conformation- } \\
\text { al change }\end{array}$ & fluorescence & $\begin{array}{l}\text { BSA } \\
\text { LEA protein }\end{array}$ & $\begin{array}{l}\text { none }^{6} \\
\text { partial }^{6}\end{array}$ \\
\hline $\begin{array}{l}\text { phosphofrukto- } \\
\text { kinase }\end{array}$ & activity & absorbance & $\begin{array}{l}\text { glucose }^{a} \\
\text { glycerol }^{a} \\
\text { maltose }^{a} \\
\text { proline }^{a} \\
\text { sucrose }^{a} \\
\text { TMAO }^{a} \\
\text { trehalose }^{a}\end{array}$ & $\begin{array}{l}\text { full }^{15} \\
\text { full }^{15} \\
\text { full } \\
\text { none } \\
\text { full } \\
\text { partial } \\
\text { full }\end{array}$ \\
\hline Pstl & activity & DNA digestion & $\begin{array}{l}\text { dextran } \\
\text { erythritol }^{a} \\
\text { Ficoll }{ }^{a}{ }^{a} \\
\text { galactitol }^{a} \\
\text { galactose }^{a} \\
\text { glucose }^{a} \\
\text { inulin }^{a} \\
\text { lactose }^{\text {a }} \\
\text { lactulose }^{a} \\
\text { maltose }^{a} \\
\text { mannitol }^{a} \\
\text { mannose }^{a} \\
\text { myo-inositol }^{a} \\
\text { palatinose }^{a} \\
\text { sorbitol }^{a} \\
\text { sucrose }^{a}\end{array}$ & $\begin{array}{l}\text { none }^{4} \\
\text { none }^{4} \\
\text { nontial }^{4} \\
\text { none } \\
\text { partial }^{4} \\
\text { none }^{4} \\
\text { partial }^{4} \\
\text { partial }^{4} \\
\text { partial }^{4} \\
\text { none }^{4} \\
\text { none }^{4} \\
\text { none }^{4} \\
\text { none }^{4} \\
\text { partial }^{4} \\
\text { partial }^{4}\end{array}$ \\
\hline
\end{tabular}




\begin{tabular}{|c|c|c|c|c|}
\hline Test Protein & Assay & Output & Additive & Protection \\
& & & threitol $^{a}$ & none $^{4}$ \\
& & & trehalose $^{a}$ & partial $^{4}$ \\
\hline Sequenase ${ }^{\circledR} \quad(a$ & activity & Dideoxy chain- & trehalose $^{a}$ & partial $^{4}$ \\
modified T7 DNA & & termination & & \\
polymerase) & & sequencing & & \\
\hline T4 DNA ligase & activity & DNA ligation & trehalose & \\
\hline T-REx293 & aggregation & light scattering & dextran & partial $^{4}$ \\
proteome & & & Ficoll ${ }^{a}$ & partial $^{6}$ \\
& & & LEA protein & partial $^{6}$ \\
\end{tabular}

${ }^{\text {aReferences }}{ }^{4,15}$ used air-dried samples. 
Table S2. Ability of additives to protect proteins against freeze-thaw stress.

\begin{tabular}{|c|c|c|c|c|}
\hline & Assay & Output & Additive & Protection \\
\hline $\begin{array}{l}\text { ADP-glucose- } \\
\text { pyrophosphorylase }\end{array}$ & activity & absorbance & $\begin{array}{l}\beta \text {-lactoglobulin } \\
\text { LEA protein } \\
\text { ribonuclease A }\end{array}$ & $\begin{array}{l}\text { none }^{1} \\
\text { partial }^{1} \\
\text { none }^{1}\end{array}$ \\
\hline $\begin{array}{c}\text { alcohol } \\
\text { dehydrogenase }\end{array}$ & activity & absorbance & $\begin{array}{l}\text { BSA } \\
\text { glutamate }\end{array}$ & $\begin{array}{l}\text { none }^{16} \\
\text { partial }^{16}\end{array}$ \\
\hline catalase & activity & absorbance & $\begin{array}{l}\text { dextran } \\
\text { dimethyl sulfoxide } \\
\text { glycerol } \\
\text { polyvinylpyrrolidone }\end{array}$ & $\begin{array}{l}\text { full }^{17} \\
\text { full }^{17} \\
\text { partial }^{17} \\
\text { full }^{17}\end{array}$ \\
\hline citrate synthase & $\begin{array}{l}\text { activity } \\
\text { aggregation }\end{array}$ & \begin{tabular}{l|} 
absorbance \\
light scattering
\end{tabular} & $\begin{array}{l}\text { BSA } \\
\text { LEA protein } \\
\text { BSA } \\
\text { LEA protein }\end{array}$ & $\begin{array}{l}\text { full }^{5} \\
\text { full }^{5} \\
\text { partial }^{5} \\
\text { full }{ }^{5,6}\end{array}$ \\
\hline $\begin{array}{l}\text { glucose-6- } \\
\text { phosphate } \\
\text { dehydrogenase }\end{array}$ & activity & absorbance & $\begin{array}{l}\beta \text {-lactoglobulin } \\
\text { BSA } \\
\text { glutamate } \\
\text { LEA protein } \\
\text { ribonuclease A }\end{array}$ & $\begin{array}{l}\text { none }^{1} \\
\text { partial }^{16} \\
\text { none }^{16} \\
\text { partial }^{1} \\
\text { none }^{1}\end{array}$ \\
\hline $\begin{array}{c}\text { lactate } \\
\text { dehydrogenase }\end{array}$ & activity & absorbance & $\begin{array}{l}\alpha \text {-crystallin } \\
\text { apo-transferrin } \\
\beta \text {-lactoglobulin } \\
\beta \text {-cyclodextrin } \\
\text { betaine }\end{array}$ & $\begin{array}{l}\text { partial }^{18} \\
\text { partial }^{19} \\
\text { partial }^{1,19-21} \\
\text { none }^{22} \\
\text { none }^{23} \text { partial }^{24,25}\end{array}$ \\
\hline
\end{tabular}




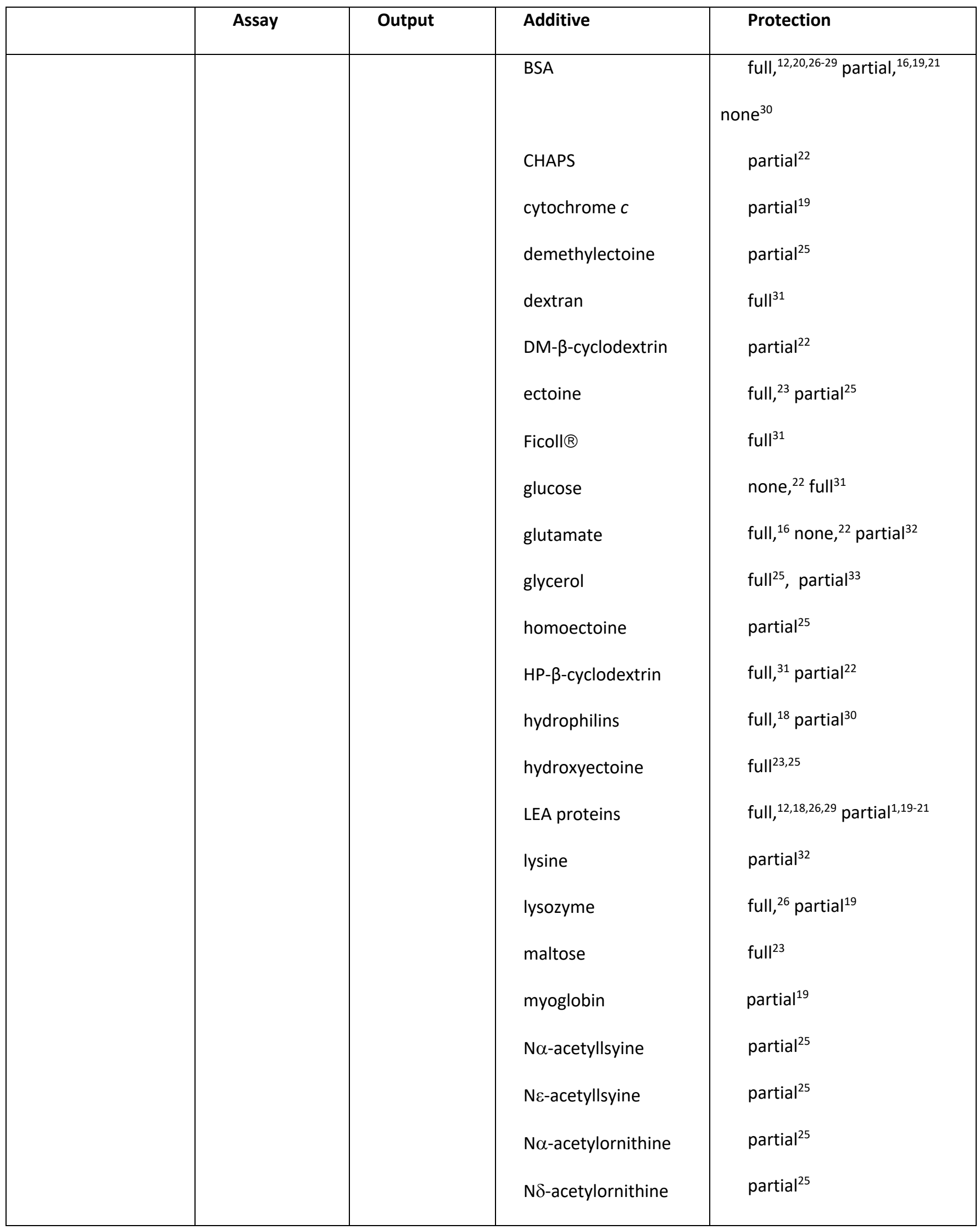




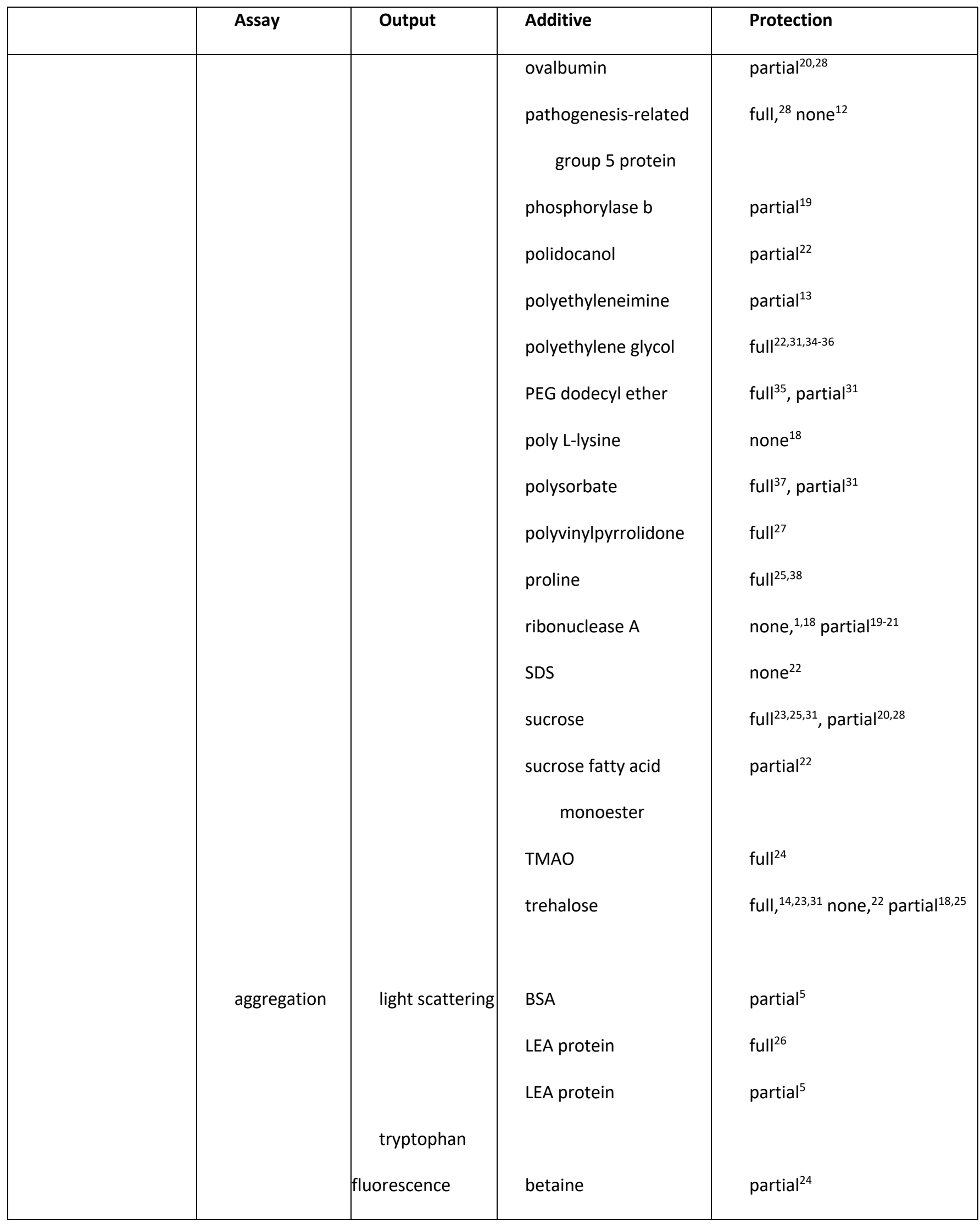




\begin{tabular}{|c|c|c|c|c|}
\hline & Assay & Output & Additive & Protection \\
\hline & $\begin{array}{r}\text { structural } \\
\text { perturbation }\end{array}$ & & $\begin{array}{l}\text { ectoine } \\
\text { hydroxyectoine } \\
\mathrm{N} \alpha \text {-acetyllsyine } \\
\mathrm{N} \varepsilon \text {-acetyllsyine } \\
\mathrm{N} \alpha \text {-acetylornithine } \\
\mathrm{N} \delta \text {-acetylornithine } \\
\text { proline } \\
\text { sucrose } \\
\text { TMAO } \\
\text { trehalose }\end{array}$ & $\begin{array}{l}\text { full }^{24} \\
\text { full }^{24} \\
\text { full }^{24} \\
\text { full }^{24} \\
\text { full }^{24} \\
\text { full } \\
\text { partial } \\
\text { full } \\
\text { full }\end{array}$ \\
\hline lipoprotein lipase & activity & fluorescence & $\begin{array}{l}\text { BSA } \\
\text { CAHS protein } \\
\text { trehalose }\end{array}$ & $\begin{array}{l}\text { partial }^{10} \\
\text { full }^{10} \\
\text { none }^{10}\end{array}$ \\
\hline $\begin{array}{c}\text { malate } \\
\text { dehydrogenase }\end{array}$ & activity & absorbance & $\begin{array}{l}\text { BSA } \\
\text { glutamate }\end{array}$ & $\begin{array}{l}\text { partial }^{16} \\
\text { partial }^{16}\end{array}$ \\
\hline $\begin{array}{l}\text { phosphofructo- } \\
\text { kinase }\end{array}$ & activity & absorbance & $\begin{array}{l}\text { betaine } \\
\text { ectoine } \\
\text { hydroxyectoine } \\
\text { maltose } \\
\text { polyethylene glycol } \\
\text { sucrose } \\
\text { trehalose }\end{array}$ & $\begin{array}{l}\text { full } \\
\text { full }^{23} \\
\text { full }^{23} \\
\text { partial } \\
\text { full } \\
\text { full } \\
\text { full }\end{array}$ \\
\hline pyruvate kinase & activity & absorbance & $\begin{array}{l}\text { BSA } \\
\text { glutamate }\end{array}$ & $\begin{array}{l}\text { partial }^{16} \\
\text { partial }^{16}\end{array}$ \\
\hline
\end{tabular}


Table S3. Ability of additives to protect against freeze-drying.

\begin{tabular}{|c|c|c|c|c|}
\hline Test Protein & Assay & Output & Additive & Protection \\
\hline $\begin{array}{c}\text { alkaline } \\
\text { phosphatase }\end{array}$ & activity & absorbance & $\begin{array}{l}\text { lactose } \\
\text { mannitol } \\
\text { trehalose }\end{array}$ & $\begin{array}{l}\text { full }^{39} \\
\text { none } \\
\text { full }^{39}\end{array}$ \\
\hline ATPase & activity & absorbance & $\begin{array}{l}\text { skim milk } \\
\text { sucrose } \\
\text { trehalose }\end{array}$ & $\begin{array}{l}\text { none }^{40} \\
\text { none }{ }^{40} \\
\text { full }^{40}\end{array}$ \\
\hline $\begin{array}{c}\beta- \\
\text { galactosidase }\end{array}$ & activity & absorbance & $\begin{array}{l}\alpha \text {-cyclodextrin } \\
\beta \text {-cyclodextrin } \\
\gamma \text {-cyclodextrin } \\
\text { CHAPS } \\
\text { DM- } \beta \text {-cyclodextrin } \\
\text { glucose } \\
\text { glutamate } \\
\text { HP- } \beta \text {-cyclodextrin } \\
\text { polidocanol } \\
\text { polyethylene glycol } \\
\text { proline } \\
\text { SDS } \\
\text { sucrose fatty acid } \\
\text { monoester } \\
\text { trehalose }\end{array}$ & $\begin{array}{l}\text { none }^{41} \\
\text { none }^{41} \\
\text { partial }^{41} \\
\text { partial }\left.\right|^{41} \\
\text { full }{ }^{41} \\
\text { full }{ }^{41} \\
\text { partial } \\
\text { none }{ }^{41} \\
\text { partial }^{41} \\
\text { full }^{41} \\
\text { none } \\
\text { partial }\end{array}$ \\
\hline catalase & activity & absorbance & $\begin{array}{l}\text { alanine } \\
\text { arginine }\end{array}$ & $\begin{array}{l}\text { full }^{42} \\
\text { partial }^{42}\end{array}$ \\
\hline
\end{tabular}




\begin{tabular}{|c|c|c|c|c|}
\hline & & & $\begin{array}{l}\text { dimethyl } \\
\text { formamide } \\
\text { dimethyl sulfoxide } \\
\text { cysteine } \\
\text { glycine } \\
\text { histidine } \\
\text { lysine } \\
\text { polyvinyl- } \\
\text { pyrrolidone } \\
\text { serine } \\
\text { threonine } \\
\text { valine } \\
\text { 4-hydroxyproline }\end{array}$ & $\begin{array}{l}\text { none }^{42} \\
\text { none }^{42} \\
\text { none } \\
\text { full } \\
\text { full } \\
\text { full } \\
\text { full } \\
\text { f2 } \\
\text { full } \\
\text { full } \\
\text { none } \\
\text { full }\end{array}$ \\
\hline $\begin{array}{c}\mathrm{L}- \\
\text { asparaginase }\end{array}$ & $\begin{array}{l}\text { activity } \\
\text { aggrega- } \\
\text { tion }\end{array}$ & absorbance & $\begin{array}{l}\text { glucose } \\
\text { lactose } \\
\text { maltose } \\
\text { mannitol } \\
\text { sucrose } \\
\text { trehalose } \\
\text { glucose } \\
\text { lactose } \\
\text { maltose } \\
\text { mannitol } \\
\text { sucrose } \\
\text { trehalose }\end{array}$ & 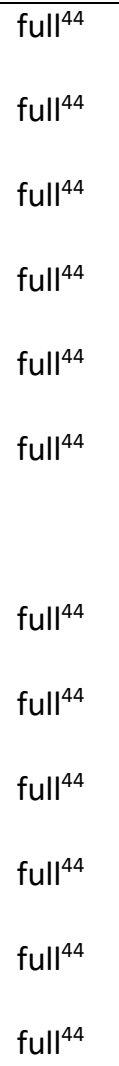 \\
\hline
\end{tabular}




\begin{tabular}{|c|c|c|c|c|}
\hline $\begin{array}{c}\text { lactate } \\
\text { dehydrogenase }\end{array}$ & activity & absorbance & $\begin{array}{l}\alpha \text {-cyclodextrin } \\
\beta \text {-cyclodextrin } \\
\gamma \text {-cyclodextrin } \\
\text { betaine } \\
\text { BSA } \\
\text { CHAPS } \\
\text { dimethylthetin } \\
\text { DM- } \beta \text {-cyclodextrin } \\
\text { ectoine } \\
\text { glucose } \\
\text { glutamate } \\
\text { glycerol } \\
\text { polyvinyl- } \\
\text { homodeanol } \\
\text { polidone } \\
\text { betaine } \\
\text { mP- } \beta \text {-cyclodextrin } \\
\text { hydroxyectoine } \\
\text { lactose } \\
\text { maltose }\end{array}$ & 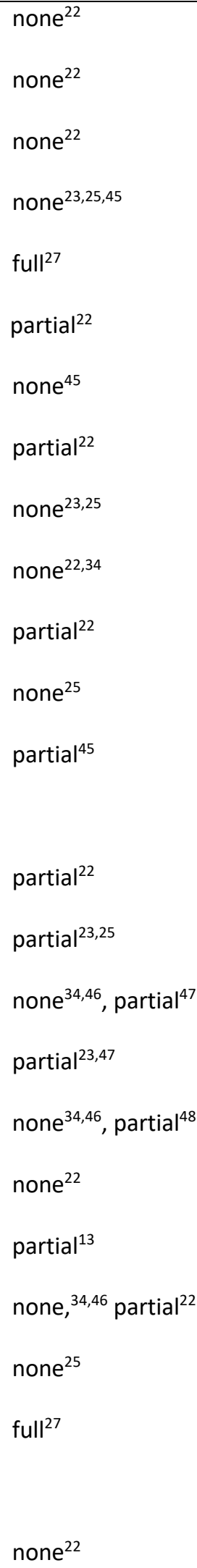 \\
\hline
\end{tabular}




\begin{tabular}{|c|c|c|c|c|}
\hline & & & $\begin{array}{l}\text { skim milk } \\
\text { sorbitol } \\
\text { sucrose } \\
\text { sucrose fatty acid } \\
\text { monoester } \\
\text { trehalose }\end{array}$ & $\begin{array}{c}\text { partial }^{40} \\
\text { partial }^{45} \\
\text { full, }^{40} \\
\text { partial }^{23,25,47,48} \\
\text { full }^{22} \\
\text { none }^{34,46} \\
\text { partial }^{22,23,25,40,45,47}\end{array}$ \\
\hline lipase & activity & $\begin{array}{c}\text { pH } \quad(\mathrm{NaOH} \\
\text { titration) }\end{array}$ & $\begin{array}{l}\text { betaine } \\
\text { dimethylthetin } \\
\text { homodeanol } \\
\text { betaine } \\
\text { sorbitol } \\
\text { trehalose }\end{array}$ & $\begin{array}{l}\text { none }^{45} \\
\text { partial| }^{45} \\
\text { none }^{45} \\
\text { none }{ }^{45} \\
\text { partial }^{45}\end{array}$ \\
\hline $\begin{array}{l}\text { lipoprotein } \\
\text { lipase }\end{array}$ & activity & fluorescence & $\begin{array}{l}\text { BSA } \\
\text { CAHS protein } \\
\text { trehalose }\end{array}$ & $\begin{array}{l}\text { partial }^{10} \\
\text { full }^{10} \\
\text { none }^{10}\end{array}$ \\
\hline $\begin{array}{c}\text { mannitol } \\
\text { dehydrogenase }\end{array}$ & activity & absorbance & $\begin{array}{l}\text { glucosylglycerate } \\
\text { glucosylglycerol } \\
\text { trehalose }\end{array}$ & $\begin{array}{l}\text { partial }^{49} \\
\text { full }^{49} \\
\text { partial }^{49}\end{array}$ \\
\hline $\begin{array}{l}\text { monoclonal } \\
\text { antibodies }\end{array}$ & $\begin{array}{l}\text { aggrega- } \\
\text { tion }\end{array}$ & light scattering & sucrose & full $^{50}$ \\
\hline $\begin{array}{l}\text { phosphor- } \\
\text { fructokinase }\end{array}$ & activity & absorbance & $\begin{array}{l}\text { betaine } \\
\text { ectoine } \\
\text { hydroxyectoine } \\
\text { glucose }\end{array}$ & $\begin{array}{l}\text { partial }^{23,25} \\
\text { partial }^{23,25} \\
\text { partial }^{23,25} \\
\text { none }^{34,46}\end{array}$ \\
\hline
\end{tabular}




\begin{tabular}{|l|l|l|c|}
\hline \multirow{2}{*}{} & & glycerol & none $^{25}$ \\
& maltose & partial $^{23}$ \\
& mannitol & none $^{34,46}$ \\
& polyethylene glycol & none $^{34,46}$ \\
& proline & none $^{25}$ \\
& sucrose & partial $^{23,25}$ \\
& trehalose & none, $^{23,34,46}$ \\
& & & partial $^{15,25}$ \\
\hline
\end{tabular}

${ }^{a}$ References ${ }^{34,46}$ combine one project. 
Table S4. Comparing excipients that protect lactate dehydrogenase (LDH)

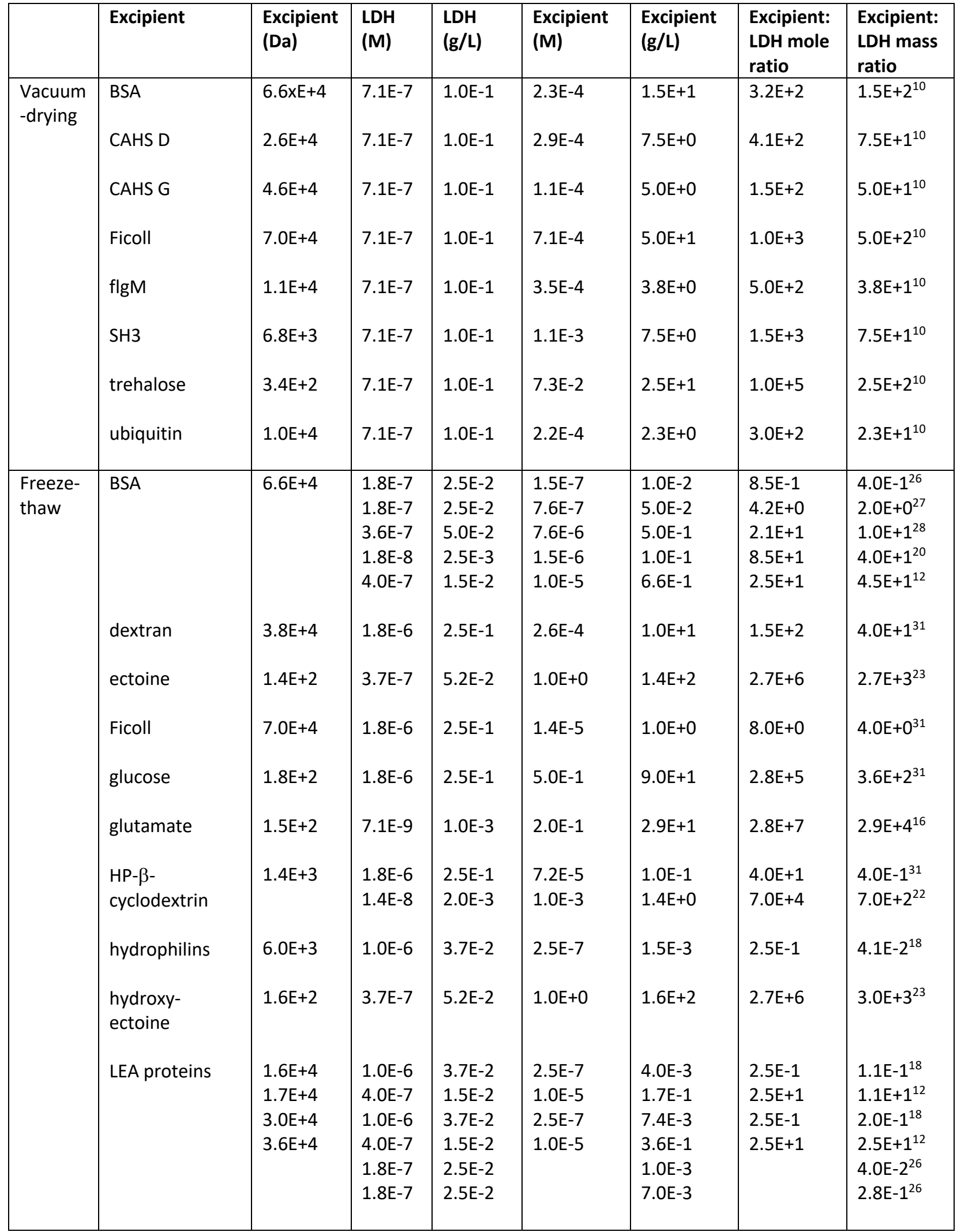




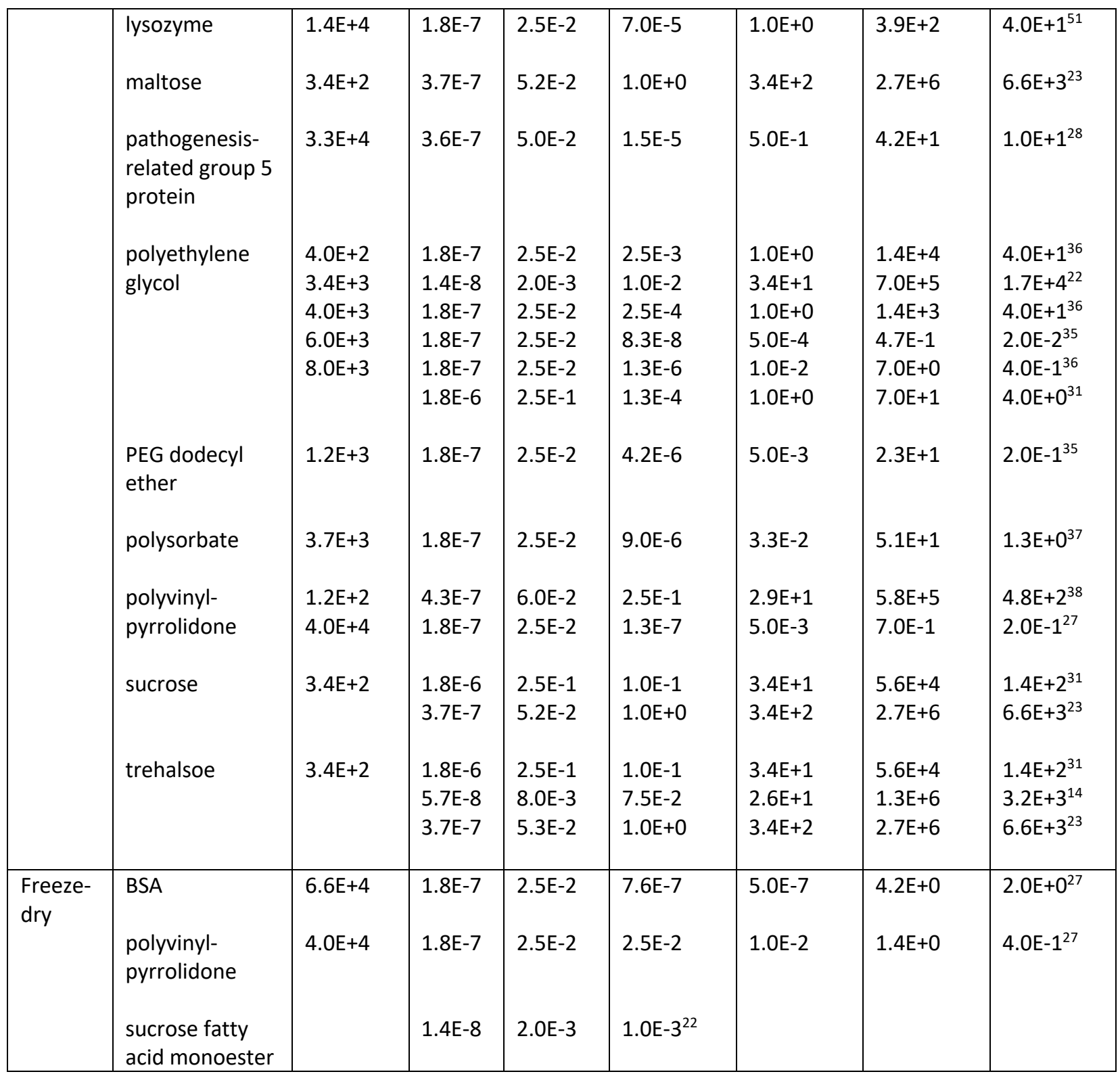




\section{REFERENCES}

(1) Popova, A. V., Rausch, S., Hundertmark, M., Gibon, Y., and Hincha, D. K. (2015) The intrinsically disordered protein lea7 from Arabidopsis thaliana protects the isolated enzyme lactate dehydrogenase and enzymes in a soluble leaf proteome during freezing and drying. Biochim. Biophys. Acta 1854, 1517-1525.

(2) Chakrabortee, S., Boschetti, C., Walton, L. J., Sarkar, S., Rubinsztein, D. C., and Tunnacliffe, A. (2007) Hydrophilic protein associated with desiccation tolerance exhibits broad protein stabilization function. Proc. Natl. Acad. Sci. USA 104, 18073-18078.

(3) Uritani, M., Takai, M., and Yoshinaga, K. (1995) Protective effect of disaccharides on restriction endonucleases during drying under vacuum. $J$. Biochem. 117, 774-779.

(4) Colaco, C., Sen, S., Thangavelu, M., Pinder, S., and Roser, B. (1992) Extraordinary stability of enzymes dried in trehalose: Simplified molecular biology. Biotechnology 10, 1007-1011.

(5) Goyal, K., Walton, L. J., and Tunnacliffe, A. (2005) LEA proteins prevent protein aggregation due to water stress. Biochem. J. 388, 151-157.

(6) Chakrabortee, S., Tripathi, R., Watson, M., Schierle, G. S., Kurniawan, D. P., Kaminski, C. F., Wise, M. J., and Tunnacliffe, A. (2012) Intrinsically disordered proteins as molecular shields. Mol. BioSyst. 8, 210-219.

(7) Rossi, S., Buera, M. P., Moreno, S., and Chirife, J. (1997) Stabilization of the restriction enzyme ecori dried with trehalose and other selected glass-forming solutes. Biotechnol. Prog. 13, 609-616.

(8) Grelet, J., Benamar, A., Teyssier, E., Avelange-Macherel, M. H., Grunwald, D., and Macherel, D. (2005) Identification in pea seed mitochondria of a lateembryogenesis abundant protein able to protect enzymes from drying. Plant Physiol. 137, 157-167.

(9) Reyes, J. L., Rodrigo, M. J., Colmenero-Flores, J. M., Gil, J. V., Garay-Arroyo, A., Campos, F., Salamini, F., Bartels, D., and Covarrubias, A. A. (2005) Hydrophilins from distant organisms can protect enzymatic activities from water limitation effects in vitro. Plant Cell Environ. 28, 709-718.

(10) Piszkiewicz, S., Gunn, K. H., Warmuth, O., Propst, A., Mehta, A., Nguyen, K. H., Kuhlman, E., Guseman, A. J., Stadmiller, S. S., Boothby, T. C., Neher, S. B., and Pielak, G. J. (2019) Protecting activity of desiccated enzymes. Protein Sci. 28, 941-951.

(11) Boothby, T. C., Tapia, H., Brozena, A. H., Piszkiewicz, S., Smith, A. E., Giovannini, I., Rebecchi, L., Pielak, G. J., Koshland, D., and Goldstein, B. (2017) Tardigrades use intrinsically disordered proteins to survive desiccation. Mol. Cell 65, 975-984 e975.

(12) Dang, N. X., Popova, A. V., Hundertmark, M., and Hincha, D. K. (2014) Functional characterization of selected LEA proteins from Arabidopsis thaliana in yeast and in vitro. Planta 240, 325-336.

(13) Andersson, M. A., and Hatti-Kaul, R. (1999) Protein stabilising effect of polyethyleneimine. J. Biotechnol. 72, 21-31. 
(14) Miller, D. P., Anderson, R. E., and de Pablo, J. J. (1998) Stabilization of lactate dehydrogenase following freeze thawing and vacuum-drying in the presence of trehalose and borate. Pharm. Res. 15, 1215-1221.

(15) Carpenter, J. F., and Crowe, J. H. (1988) Modes of stabilization of a protein by organic solutes during desiccation. Cryobiology 25, 459-470.

(16) Tamiya, T., Okahashi, N., Sakuma, R., Aoyama, T., Akahane, T., and Matsumoto, J. J. (1985) Freeze denaturation of enzymes and its prevention with additives. Cryobiology 22, 446-456.

(17) Ashwood-Smith, M. J., and Warby, C. (1972) Protective effect of low and high molecular weight compounds on the stability of catalase subjected to freezing and thawing. Cryobiology 9, 137-140.

(18) Reyes, J. L., Campos, F., Wei, H., Arora, R., Yang, Y. I., Karlson, D. T., and Covarrubias, A. A. (2008) Functional dissection of hydrophilins during in vitro freeze protection. Plant Cell Environ. 31, 1781-1790.

(19) Kazuoka, T., and Oeda, K. (1994) Purification and characterization of COR85oligomeric complex from cold-acclimated spinach. Plant Cell Physiol. 35, 601611.

(20) Lin, C. T., and Thomashow, M. F. (1992) A cold-regulated Arabidopsis gene encodes a polypeptide having potent cryoprotective activity. Biochem. Biophys. Res. Commun. 183, 1103-1108.

(21) Thalhammer, A., Bryant, G., Sulpice, R., and Hincha, D. K. (2014) Disordered cold regulated 15 proteins protect chloroplast membranes during freezing through binding and folding, but do not stabilize chloroplast enzymes in vivo. Plant Physiol. 166, 190-201.

(22) Izutsu, K., Yoshioka, S., and Terao, T. (1994) Stabilizing effect of amphiphilic excipients on the freeze-thawing and freeze-drying of lactate dehydrogenase. Biotechnol. Bioeng. 43, 1102-1107.

(23) Lippert, K., and Galinski, E. A. (1992) Enzyme stabilization by ectoine-type compatible solutes - protection against heating, freezing and drying. Appl. Microbiol. Biotechnol. 37, 61-65.

(24) Goller, K., and Galinski, E. A. (1999) Protection of a model enzyme (lactate dehydrogenase) against heat, urea and freeze-thaw treatment by compatible solute additives. J. Mol. Catal. B-Enzym. 7, 37-45.

(25) Galinski, E. A. (1993) Compatible solutes of halophilic eubacteria - molecular principles, water-solute interaction, stress protection. Experientia 49, 487-496.

(26) Hughes, S., and Graether, S. P. (2011) Cryoprotective mechanism of a small intrinsically disordered dehydrin protein. Protein Sci. 20, 42-50.

(27) Anchordoquy, T. J., and Carpenter, J. F. (1996) Polymers protect lactate dehydrogenase during freeze-drying by inhibiting dissociation in the frozen state. Arch. Biochem. Biophys. 332, 231-238.

(28) Dave, R. S., and Mitra, R. K. (1998) A low temperature induced apoplastic protein isolated from Arachis hypogaea. Phytochemistry 49, 2207-2213.

(29) Wisniewski, M., Webb, R., Balsamo, R., Close, T. J., Yu, X. M., and Griffith, M. (1999) Purification, immunolocalization, cryoprotective, and antifreeze activity of pca60: A dehydrin from peach (Prunus persica). Physiol. Plant. 105, 600-608. 
(30) Konrad, Z., and Bar-Zvi, D. (2008) Synergism between the chaperone-like activity of the stress regulated ASR1 protein and the osmolyte glycine-betaine. Planta 227, 1213-1219.

(31) Anchordoquy, T. J., Izutsu, K. I., Randolph, T. W., and Carpenter, J. F. (2001) Maintenance of quaternary structure in the frozen state stabilizes lactate dehydrogenase during freeze-drying. Arch. Biochem. Biophys. 390, 35-41.

(32) Seguro, K., Tamiya, T., Tsuchiya, T., and Matsumoto, J. J. (1990) Cryoprotective effect of sodium glutamate and lysine- $\mathrm{HCl}$ on freeze denaturation of lactatedehydrogenase. Cryobiology 27, 70-79.

(33) Soliman, F. S., and Van den Berg, L. (1971) Factors affecting freezing damage of lactic dehydrogenase. Cryobiology 8, 73-78.

(34) Carpenter, J. F., Prestrelski, S. J., and Arakawa, T. (1993) Separation of freezing- and drying-induced denaturation of lyophilized proteins using stressspecific stabilization. I. Enzyme activity and calorimetric studies. Arch. Biochem. Biophys. 303, 456-464.

(35) Hillgren, A., and Alden, M. (2002) A comparison between the protection of LDH during freeze-thawing by PEG 6000 and Brij 35 at low concentrations. Int. $J$. Pharm. 244, 137-149.

(36) Mi, Y. L., Wood, G., and Thoma, L. (2004) Cryoprotection mechanisms of polyethylene glycols on lactate dehydrogenase during freeze-thawing. AAPS J. 6.

(37) Hillgren, A., Lindgren, J., and Alden, M. (2002) Protection mechanism of Tween 80 during freeze-thawing of a model protein, LDH. Int. J. Pharm. 237, 57-69.

(38) Rajendrakumar, C. S. V., Reddy, B. V. B., and Reddy, A. R. (1994) Prolineprotein interactions - protection of structural and functional integrity of $\mathrm{M}_{4}$ lactatedehydrogenase. Biochem. Biophys. Res. Commun. 201, 957-963.

(39) Ford, A. W., and Dawson, P. J. (1993) The effect of carbohydrate additives in the freeze-drying of alkaline phosphatase. J. Pharm. Pharmacol. 45, 86-93.

(40) Li, B., Tian, F., Liu, X., Zhao, J., Zhang, H., and Chen, W. (2011) Effects of cryoprotectants on viability of Lactobacillus reuteri CICC6226. Appl. Microbiol. Biotechnol. 92, 609-616.

(41) Izutsu, K., Yoshioka, S., and Terao, T. (1993) Stabilization of $\beta$-galactosidase by amphiphilic additives during freeze-drying. Int. J. Pharm. 90, 187-194.

(42) Lale, S. V., Goyal, M., and Bansal, A. K. (2011) Development of lyophilization cycle and effect of excipients on the stability of catalase during lyophilization. Int. J. Pharm. Invest. 1, 214-221.

(43) Darbyshire, B. (1974) The influence of dehydration on catalase stability. A comparison with freezing effects. Cryobiology 11, 148-151.

(44) Ward, K. R., Adams, G. D. J., Alpar, H. O., and Irwin, W. J. (1999) Protection of the enzyme L-asparaginase during lyophilisation - a molecular modelling approach to predict required level of lyoprotectant. Int. J. Pharm. 187, 153-162.

Vasudevamurthy, M. K., Weatherley, L. R., and Lever, M. (2005) Enzyme stabilization using synthetic compensatory solutes. Biocatal. Biotransfor. 23, 285291.

(46) Prestrelski, S. J., Arakawa, T., and Carpenter, J. F. (1993) Separation of freezing-induced and drying-induced denaturation of lyophilized proteins using 
stress-specific stabilization .2. Structural studies using infrared-spectroscopy. Arch. Biochem. Biophys. 303, 465-473.

(47) Kawai, K., and Suzuki, T. (2007) Stabilizing effect of four types of disaccharide on the enzymatic activity of freeze-dried lactate dehydrogenase: Step by step evaluation from freezing to storage. Pharm. Res. 24, 1883-1890.

(48) Izutsu, K., and Kojima, S. (2002) Excipient crystallinity and its protein-structurestabilizing effect during freeze-drying. J. Pharm. Pharmacol. 54, 1033-1039.

(49) Sawangwan, T., Goedl, C., and Nidetzky, B. (2010) Glucosylglycerol and glucosylglycerate as enzyme stabilizers. Biotechnol. J. 5, 187-191.

(50) Shire, S. J. (2009) Formulation and manufacturability of biologics. Curr. Opin. Biotechnol. 20, 708-714.

(51) Hughes, S. L., Schart, V., Malcolmson, J., Hogarth, K. A., Martynowicz, D. M., Tralman-Baker, E., Patel, S. N., and Graether, S. P. (2013) The importance of size and disorder in the cryoprotective effects of dehydrins. Plant Physiol. 163, 1376. 